\title{
DANIELI TECHNOLOGICAL PACKAGES LIFE CYCLE PARTNER FOR SLAB CASTER INDUSTRY*
}

Maurizio Massimo ${ }^{1}$ Mike Knights ${ }^{2}$

\begin{abstract}
Danieli developed the " $3 Q$ " suite of process control technologies to address three critical principles: delivering the highest product Quality, in the required Quantity, with Quickness of response required for today's casting machines. Ever increasing market demands for productivity and slab quality necessitate the latest advances/improvements in equipment design, process technology, and control software for all types of slab casters, and Danieli boasts a unique experience that extends from ultra high-speed thin slab casters to extra high-thickness conventional slab casters. The " $3 Q$ " system is monitored and controlled by a unique, intelligent $\mathrm{HMI}$ incorporating. Touch screen technology and Operator Assistant tablets. Operator Assistant is a tablet based system that guides the operator through the plant process, so that in the event of a process change or an alarm the operator is automatically presented with the choice of possible solutions / actions. The comprehensive range of technological packages cover all aspects of caster design and automation control, from feeding of liquid steel into the tundish to the final slab leaving the caster.
\end{abstract}

Keywords: Continuous slab caster; 3Q; Technology packages; Process technology; High-speed casting; Automation.

1 Engineer Sales Director Danieli Davy Distington, Danieli \& C. Officine Meccaniche S.p.A, Buttrio, UD, Italy.

2 Executive Vice President Sales Danieli Davy Distington, Danieli \& C. Officine Meccaniche S.p.A, Buttrio, UD, Italy. 


\section{INTRODUCTION}

With increasing competition in the worldwide steel market, continuous casting lines are under pressure to optimise production, produce new steels with higher added value and at the same time, cut costs. This paper describes some of these technological advances that have been developed to allow high-speed thin slab casting (up to $8 \mathrm{~m} / \mathrm{min}$ ) and how this technology is adapted and applied to "conventional" thickness slab casters.

To allow the global steel maker to achieve these objectives, Danieli has developed a Human Computer Interface $(\mathrm{HCl})$ and a suite of " $3 Q$ " technologies based on their wealth of experience and knowledge in continuous casting.

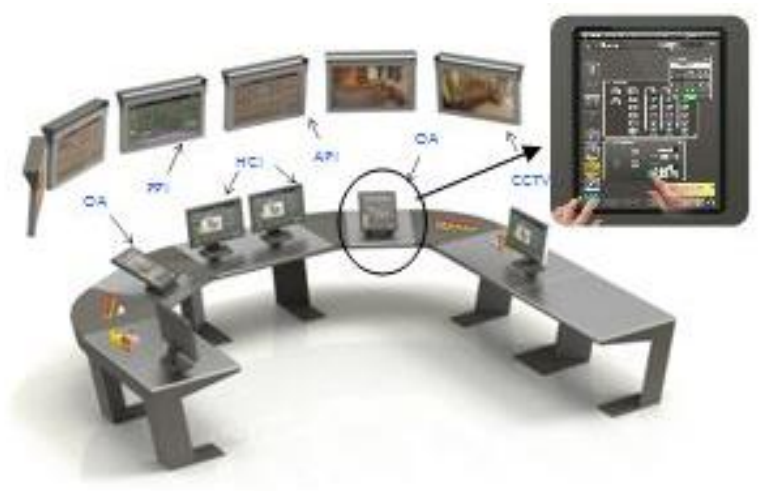

Figure 1 - Typical HCl Control "Cockpit"

\section{Human Computer Interface $(\mathrm{HCl})$}

The "3Q" system is monitored and controlled by a unique intelligent Human Computer Interface (Figure 1), incorporating "touch screen" technology.

The Plant Performance Indicator (PPI) provides information on the various processes for the overall steel plant and is orientated towards plant management.

The Area Performance Indicator (API) provides overall information on the various caster processes and is orientated towards the caster operator / technologist.

The Operator Assistant (OA), Figure 2, is a tablet-based system which guides the operator through the plant process, such that in the event of a process change or an alarm, the operator is automatically presented with the choice of possible solutions / actions.

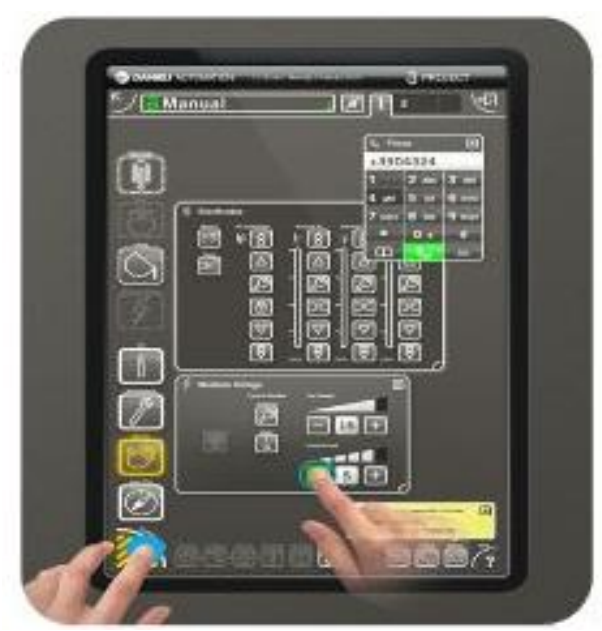

Figure 2 - Operator Assistant (OA) 


\section{“3Q” Technological Packages}

The "3Q" suite of technological packages covers all aspects of caster design and control from liquid steel feeding to discharging of the final slab. The following selection from more than twelve (12) " $3 Q$ " technology packages is considered in this paper:

Q-LEVEL+ Mould level control system

Q-MAP Real-time mould monitoring system

Q-WIDTH Mould width changing technology

Q-INMO+ Hydraulic mould oscillator technology

Q-COOL Dynamic secondary cooling control

Q-CORE Dynamic soft reduction (DSR)

\section{Q-LEVEL+}

Q-LEVEL+ is a mould level control system specifically developed to improve the steady state condition of liquid steel flow into the mould by counteracting any adverse mould level disturbances, thus promoting slab surface and internal quality.

Q-LEVEL+ is composed of three modules with specific and dedicated functions:

> An optimal Controller composed of a Linear Quadratic Estimator (LQE), to analyse the real time process data and a LQR (Linear Quadratic Regulator), both making it possible to change the controller mode and increase or decrease the speed of response.

> Supervision and Control strategies are always active and determine the correct strategy to be utilised for the current casting condition.

> Special filter systems are employed to counteract phenomena such as dynamic bulging

A major innovation of the system is the ability to analyse and process level fluctuation due to a multitude of periodic disturbances, such as dynamic bulging or roll eccentricity. The process sequencing is as follows: -

$>$ A Fast Fourier Transfer (FFT) analysis is performed on the level fluctuation.

$>$ Identification of a specific disturbance frequency and amplitude is performed.

$>$ The anticipated flow control correction is determined and a counteracting signal injected into the control stopper/slidegate.

Figure 3 illustrates a mould level trend (red plot) and stopper feed forward (f.f.) action (blue plot). The left hand side shows the mould level variation with NO stopper f.f. where a periodic disturbance has been identified. The right hand side shows the improved mould level variation when stopper f.f. correction is active. In this example, the level trend standard deviation reduces from 1.74 to $1.01 \mathrm{~mm}$, an improvement of $72 \%$.

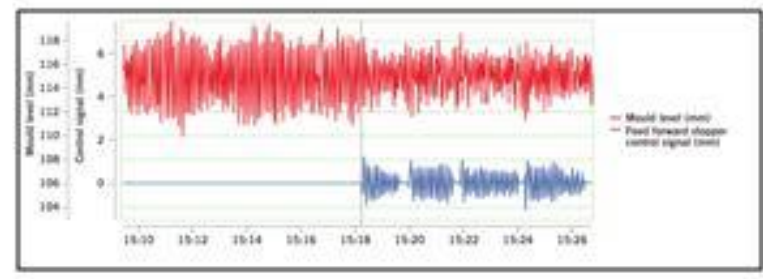

Figure 3 - Mould Level Trace With and Without Feed Forward Stopper Control Active 
The system regulator is a new generation, compared with traditional PID controllers. Q-LEVEL+ has the ability to analyse the level behaviour, anticipate the disturbance and take the appropriate preventative action, providing a faster reaction time and guaranteeing a greater mould level stability.

\section{Q-MAP}

Q-MAP is a thermocouple-based real-time mould monitoring system. By inserting a network of thermocouples in the mould copper plates, abnormal solidification conditions in the mould can be identified, such as shell sticking to the copper plates.

The signals from the thermocouples are constantly monitored and collected by a PLC and sent to the technological work station, where the signals are processed according to Q-MAP algorithms and then displayed. When a breakout situation is developing, Figure 4, a warning is given and corrective actions are sent to the PLC to perform the following sequence:

$>$ Taper correction

$>$ Casting speed reduction

$>$ Speed stoppage

When steady state mould conditions are established, normal casting is resumed.

The thermal monitoring function is capable of generating a graphical and numerical "map" occurring within the mould, which can be used to monitor performance such as mould lubrication, heat transfer and solidification conditions.

This feature becomes important for high-speed thin slab casting (up to $8 \mathrm{~m} / \mathrm{min}$ ) where fast reaction times are essential and can only be achieved by the automation system. Figure 5 shows a thermal map when casting at $4.4 \mathrm{~m} / \mathrm{min}$.

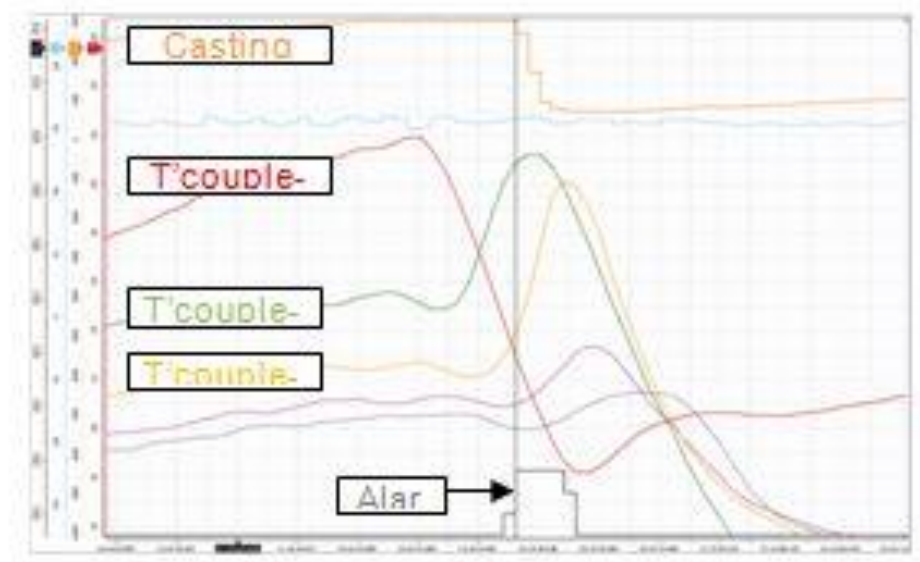

Figure 4 - Mould Thermocouple Traces during a Breakout Alarm Condition

All the thermocouple temperatures are recorded on a PC providing an historical log of events, which can use Q-MAP tools off-line to analyse casting trends, such as mould lubrication or SEN performance. 


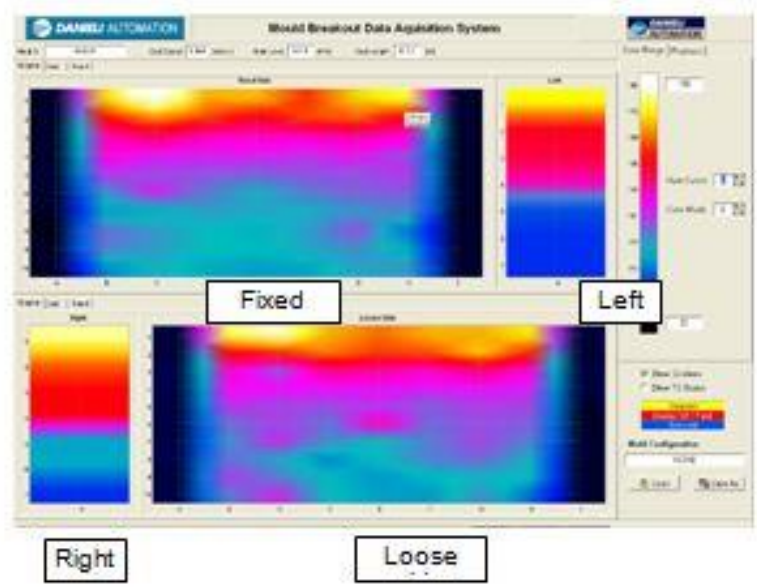

Figure 5 - Thermal Map of Each Copper Plate

\section{Q-WIDTH}

Q-WIDTH is advanced electro-mechanical mould width changing technology, combining mechanical and software solutions that allow on-line width changing (increasing and decreasing) without interrupting production. Movement of the narrow side copper plate assemblies is achieved by 4 high-capacity satellite roller screw jacks (figure 6) mounted within the mould assembly. Each jack is driven by a servo motor, which can be mounted on or off the mould, via a gearbox mounted on the end of the jack. To achieve concise positioning of the narrow side assembly, a transducer inside the screw jack directly measures position, thus eliminating backlash and accurate encoders mounted on the gearbox are also utilised.

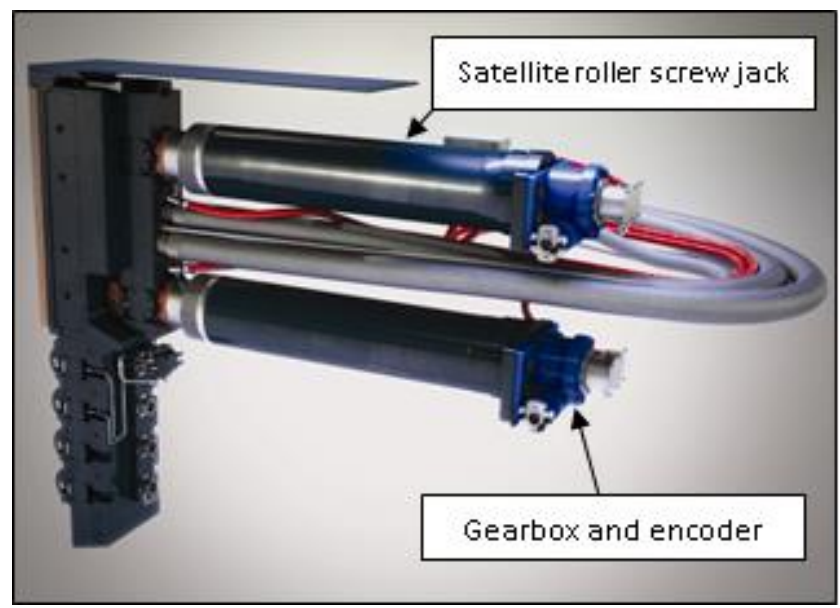

Figure 6 - Q-WIDTH Assembly

During normal casting, the system has the capability to continuously adjust the narrow side taper according to changing casting conditions. During width changing, the control software will calculate, in real time, the optimum narrow side taper to avoid an "air gap" forming between the solidifying shell and the copper plate, thus minimising the possibility of "breakouts" and at the same time, maintaining slab shape and quality.

The system is equipped with a stationary braking device which guarantees the position is maintained, even during an electrical failure, which is not the case with the alternative hydraulically actuated system. 
A typical width changing operation is shown in figure 7:

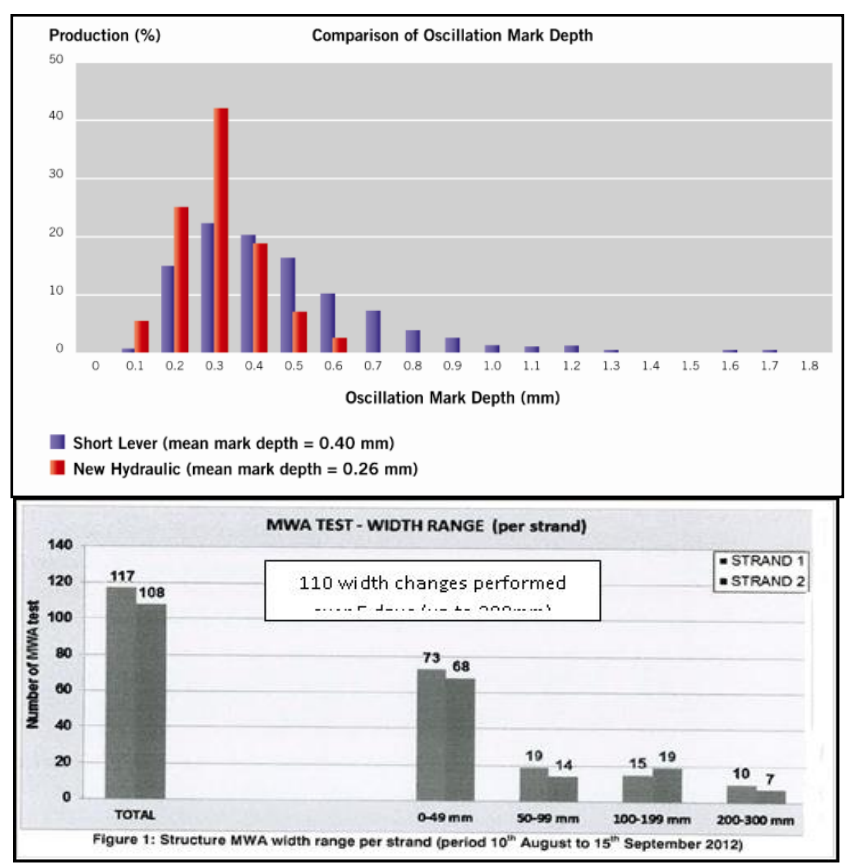

Figure 7 - Typical Width Changing Schedule Performed With Q-WIDTH

\section{Q-INMO+}

Q-INMO+ is a patented solution from DANIELI for the mould oscillation system that delivers optimum stroke, frequency and waveform, such that oscillation mark depth is minimised and mould powder consumption is optimized, thus ensuring good heat transfer in the mould and resulting in excellent slab surface quality.

The Q-INMO+ hydraulic oscillator, Figure 8, is composed of a static frame and an oscillating frame which carries the mould. Two servo valve controlled hydraulic cylinders (one either side of the mould) are fixed to the static frame and directly drive the oscillating frame. DANIELl's unique patented "rolling element" technology provides accurate and almost frictionless guidance of the oscillating frame, even at high frequencies up to 490 cycles per minute (cast speed at $8 \mathrm{~m} / \mathrm{min}$ ).

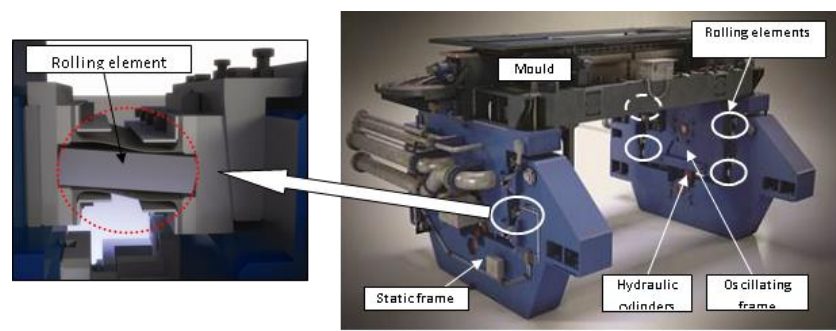

Figure 8 - Q-INMO+ Construction and Rolling Element Design

The patented "rolling element" technology is based on the principle of a roller bearing. Due to the oscillation stroke range of typically, $+/-6 \mathrm{~mm}$, only a fraction of the roller surface is necessary, therefore, only an "element" of the roller is required.

At these high frequencies and with asymmetric waveforms, the mechanical loads become very high, which have necessitated the development of the rolling element 
design to handle these demanding conditions. It is normal for a Q-INMO+ oscillator to be in operation for more than 2 years without needing a change of wearing parts. When required, the rolling elements, which are a cassette design, allow quick and easy exchange without having to remove the complete oscillator. Therefore, NO spare oscillator is required in the maintenance shop.

The hydraulic actuation of the oscillator allows online changing of the stroke, frequency and waveform (asymmetry) providing complete control of all the oscillating parameters. By optimising the negative ( $T n$ ) and positive (Tp) strip times, optimised solidification conditions in the mould are achieved to promote slab quality.

The Q-INMO+ can be retro-fitted to existing machines, which has proven to be highly successful as shown in Figure 9:

In this case, an existing electro-mechanically actuated lever-based oscillator was replaced with Q-INMO+, which dramatically reduced the oscillation mark depth to within a very tight tolerance band, reducing the need for inspection and slab rectification.

\section{Q-COOL}

Q-COOL is a dynamic secondary cooling control system utilising a dynamic "3Dimensional" mathematical cooling/solidification model, based on a finite-element algorithm. The temperature and solidifying shell thickness is calculated in real time from the meniscus to the machine exit using real time data, such as steel temperature, casting speed, mould heat extraction, etc., which are fed into the model to determine the temperature and solidification profiles, as shown in figure 9.

The calculated results are compared with aim temperature profiles stored in a database. Any deviation from the aim profile is rectified by adjusting the secondary cooling flows to achieve the optimum casting conditions, even during transient casting conditions, thus promoting consistent surface quality and a reduced frequency of surface cracks.

Q-COOL dynamically sets the water flows by independent control valves controlled in closed loops. In order to maintain elevated temperatures of the slab corner/edges (especially important in the straightening area when casting micro-alloyed steels), the system can also perform "spray width" control if this feature is installed on the caster, "Spray width" control is the ability to dynamically "turn down / off" water to wide side spray nozzles near the slab corners according to the slab width in appropriate spray zones.

Q-COOL is successfully utilised on conventional and thin slab casters, equipped with either water only or air mist secondary cooling systems.

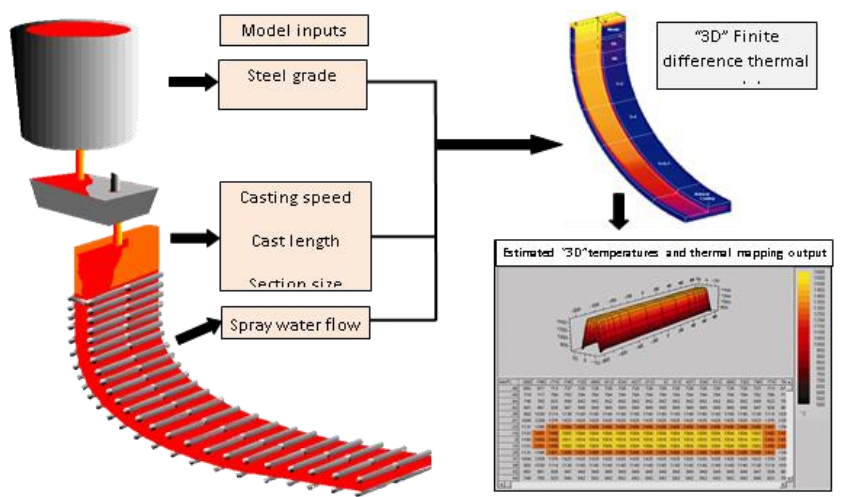

Figure 9 - Schematic Diagram of Q-COOL Model 


\section{Q-CORE}

Q-CORE is a dynamic soft reduction (DSR) system designed to reduce centreline segregation and porosity, essential for high-quality plate and line-pipe applications. By optimising roll pitch to limit slab bulging and applying DSR to reduce the internal liquid core volume, segregation can be minimised.

Soft reduction is the application of a tapered reduction in the roll gap, above the normal thermal contraction rate, at the final point of solidification. It is essential to apply the correct rate of reduction at the correct location within the machine to achieve optimum internal quality. If soft reduction is applied too early, the process becomes ineffective and segregation will still be present. If applied too late, then there is a tendency to promote internal cracking.

Q-CORE continuously monitors the solidification profile of the strand and dynamically adjusts the roll gap settings to achieve the required reduction rate (which is configurable) at the correct location within the machine.

With the correct application of DSR, centreline slab quality is assured as illustrated by the macro etch $(2000 \mathrm{~mm} \times 250 \mathrm{~mm}$ thick slab) shown in Figure 10.

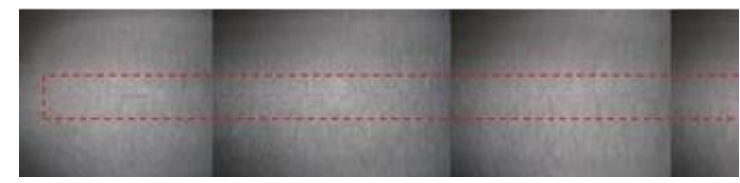

Figure 10 - Macro Etch Print of a Soft Reduced Slab with Q-CORE

The latest development of Q-CORE is the incorporation of Q-PULSE ("Patent Pending"), which is an additional function that can determine the presence of a liquid core within the machine and hence the final solidification position. By oscillating the inner radius segment frame a small amount at a given frequency, the change in liquid core volume is "transmitted" to the meniscus, where the mould level control system detects this potential movement. If a liquid core is present within the oscillated segment, then the same oscillating frequency will be seen by the mould level system, if a solid core is present, then no movement will be detected.

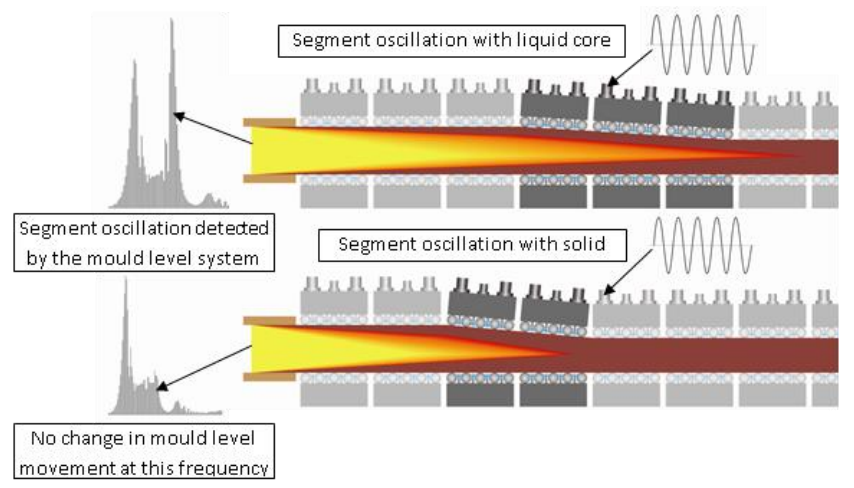

Figure 11 - Schematic Diagram Illustrating Q-PULSE

\section{CONCLUSION}

The comprehensive range of " $3 Q$ " technological packages ensures Danieli remain at the forefront of conventional and thin slab caster design, process technology and automation control. A major advantage of the package concept is they can be applied, either individually or in combination, to new machines or retro-fitted to existing casters. 